\title{
On the edge of the foreshock: model-data comparisons
}

\author{
D. G. Sibeck ${ }^{1}$, N. Omidi ${ }^{2}$, I. Dandouras ${ }^{3}$, and E. Lucek ${ }^{4}$ \\ ${ }^{1}$ Code 674, NASA/GSFC, Greenbelt, MD, USA \\ ${ }^{2}$ Solana Scientific, Solana Beach, CA, USA \\ ${ }^{3}$ CESR, Toulouse, France \\ ${ }^{4}$ Imperial College, London, UK
}

Received: 26 September 2007 - Revised: 20 March 2008 - Accepted: 16 April 2008 - Published: 11 June 2008

\begin{abstract}
We present the results of a global hybrid code simulation for the solar wind-interaction with the Earth's magnetosphere during an interval of steady radial IMF. The model predicts a foreshock marked by innumerable localized, correlated, and large amplitude, density and magnetic field strength variations, depressed velocities, and enhanced temperatures. The foreshock is bounded by a broad $\left(\sim 0.8 R_{E}\right)$ region of enhanced densities, temperatures, and magnetic field strengths that extends far $\left(\sim 8.6 R_{E}\right)$ upstream from the bow shock. Flow perturbations within the boundary are directed perpendicular to the boundary, towards the unperturbed solar wind and away from the foreshock. Cluster observations of the ion foreshock and pristine solar wind confirm the predictions of the model. The observations suggest that foreshock cavities, crater-like density and magnetic field strength structures whose cores are filled with suprathermal particles, can be interpreted in terms of transient encounters with the foreshock boundary.
\end{abstract}

Keywords. Interplanetary physics (Discontinuities; Planetary bow shocks; Plasma waves and turbulence)

\section{Introduction}

The foreshock is the region of space upstream from and magnetically connected to the bow shock that is filled with particles backstreaming from the shock (Eastwood, 2005). Electrons stream away from the bow shock, while the distributions of suprathermal ions can be classified as reflected, intermediate, or diffuse. The foreshock is the scene of intense wave-particle interactions that generate shocklets, 1 and $\sim 30$ s period waves, and accelerate the population of diffuse ions to suprathermal energies of $150 \mathrm{keV}$ or even greater.

Correspondence to: D. G. Sibeck

(david.g.sibeck@nasa.gov)
Mesoscale structures are common within and near the boundaries of the foreshock. Transient (1-10 min) bursts of suprathermal particles in the region just upstream from the bow shock are common. The bursts can frequently be associated with depressed plasma densities, magnetic field strengths, and antisunward velocities, but enhanced temperatures (Wibberenz et al., 1985; Fairfield et al., 1990). Large (factor of 2-3) density and magnetic strength increases bound these isolated events, which have come to be known as foreshock cavities. Hot flow anomalies (HFAs) exhibit similar, but far more pronounced, plasma and magnetic field variations, including flows deflected far from the Sun-Earth line (Schwartz et al., 2000). Unlike foreshock cavities, they are centered on interplanetary magnetic field (IMF) discontinuities. Sometimes events with the characteristics of HFAs can have extremely small dimensions, in which case they are known as density holes (Parks et al., 2006).

Events with the characteristics of foreshock cavities occur in local hybrid code simulations. Thomas and Brecht (1988) launched a spatially-limited beam of sunward-moving ions into the oncoming solar wind. Beam-beam instabilities thermalized the two cold particle distributions, resulting in a single heated population within a cavity bounded by density and magnetic field strength enhancements. By contrast, events with foreshock cavity characteristics have not been identified in previously reported self-consistent global hybrid code simulations (e.g. Lin, 2002, 2003; Omidi et al., 2005; Omidi, 2007). This paper presents new results from a two-dimensional self-consistent global hybrid code model for the solar wind-magnetosphere interaction during periods of steady radial IMF orientation and high Mach number. In addition to strong compressional density and magnetic field strength variations within the foreshock, the model predicts prominent density and magnetic field strength enhancements on the edge of the foreshock. We demonstrate that Cluster observations of this boundary are consistent with model predictions and argue that the previously reported foreshock

Published by Copernicus Publications on behalf of the European Geosciences Union. 


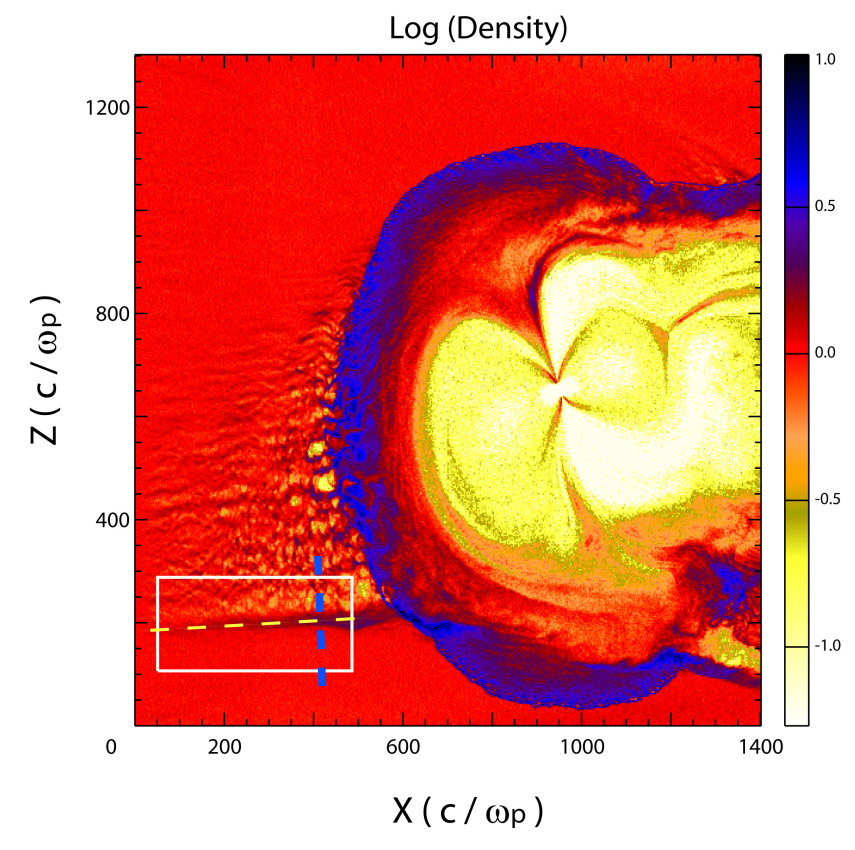

Fig. 1. Results from a hybrid code simulation in the noon-midnight meridional plane for the densities in the pristine solar wind, foreshock, magnetosheath, and magnetosphere during an interval of radial interplanetary magnetic field (IMF) orientation. The vertical line shows a cut across the sharp southern boundary of the foreshock. The horizontal line shows a cut along the sharp southern boundary of the foreshock. A box encloses a region selected for further statistical analysis and comparison with observations.

cavities can be interpreted in terms of transient encounters with the boundary of the ion foreshock.

\section{Model and predictions}

The hybrid simulation model used for this work resembles that of Omidi et al. (2004, 2005). The simulations are in the $\mathrm{X}-\mathrm{Z}$ plane where $\mathrm{X}$ points antisunward along the Sun-Earth line and $\mathrm{Z}$ points northward in the plane containing the dipole axis. Although $\partial / \partial Y=0$, the model retains all three components of the ion plasma velocity and electromagnetic fields. The solar wind plasma and electromagnetic fields enter on the sunward side of the simulation domain, while the remaining three boundaries remain open. The magnetic dipole lies at $X=950 \mathrm{c} / \omega_{p}$ and $Z=650 \mathrm{c} / \omega_{p}$, where $c / \omega_{p}$ is the proton skin depth in the solar wind. The Northern Hemisphere of the dipole tilts $20^{\circ}$ sunward. Parameter $D p$, the standoff distance of the magnetopause normalized to $c / \omega_{p}$, indicates the strength of the dipole magnetic field and is set to $D p=128$. The simulated magnetosphere is therefore 5 times smaller than the terrestrial magnetosphere $(D p=640)$. The solar wind Alfvénic Mach number is 5, with both electron and ion beta (the ratio of the kinetic to magnetic pressures) set to 0.5 . The steady IMF points radially antisunward along the positive $\mathrm{x}$-axis. The model employs a spatially uniform coefficient of resistivity corresponding to a resistive scale length of 0.3 ion skin depths, which is substantially smaller than the 1 ion skin depth cell size. Normalized values for the input solar wind densities, velocities, temperatures, and magnetic field strengths are $1,5,1$, and 1 .

By $T=50 \Omega^{-1}$, where $\Omega$ is the proton gyrofrequency in the solar wind, the dayside magnetosphere is well developed and the results are suitable for study. However, the bow shock is still expanding outward at a speed 5 times the Alfvénic velocity. Figure 1 shows model predictions for the global distribution of densities at time $T=75 \Omega^{-1}$ where $\Omega$ is the proton gyrofrequency in the solar wind. The familiar features of the solar wind-magnetosphere interaction are clearly visible: the low density (white and yellow) magnetosphere, intermediate density low latitude boundary and plasma depletion layers (red), high density magnetosheath proper (blue), and the solar wind (red). A broad region of turbulent plasma densities upstream from the bow shock at $\left(X<400 \mathrm{c} / \omega_{p}\right.$ and $\left.200<Z<800 c / \omega_{p}\right)$ defines the spatial extent of the foreshock. Within the foreshock, there are numerous density enhancements and depletions noted by Lin (2003) and described by Omidi (2007) in considerable detail.

The transition between the foreshock and the pristine solar wind is rather gradual on the northern edge of the foreshock, but quite sharp on the southern edge of the foreshock. The distinction is related to the dipole tilt: runs with no tilt produce foreshocks with sharp northern and southern edges. The vertical blue dashed line in Fig. 1 marks a north/south cut through the abrupt southern edge of the foreshock. Figure 2 presents density, total pressure (the sum of the magnetic and thermal pressures), ion temperature, ion velocity, and magnetic field strength profiles along this cut from the pristine solar wind to the turbulent foreshock. Velocities within the foreshock can be $50 \%$ less than those in the solar wind, but are typically $10-20 \%$ less. Despite the fact that magnetic field strengths and densities in the foreshock can be substantially (factor of 2) less than those in the pristine solar wind, significant (generally more than a factor of 20) enhancements in the temperature of the combined solar wind and suprathermal ion populations cause total pressures within the foreshock to greatly exceed those in the solar wind.

The imbalance between foreshock and pristine solar wind pressures means that the former region must expand at the expense of the latter. As the foreshock expands, it compresses neighboring solar wind plasma and magnetic fields, resulting in a region of enhanced solar wind densities and magnetic field strengths on the edge of the foreshock. Vertical dashed lines in Fig. 2 mark the $\sim 50 c / \omega_{p}\left(\sim 0.8 R_{E}\right)$ extent of this region. Within the edge densities can be enhanced by as much as a factor of 2 , magnetic field strengths by as much as a factor of 1.7 , temperatures by a factor ranging from 2 to 20 , and velocities are more variable and slightly lower than those in the solar wind. 


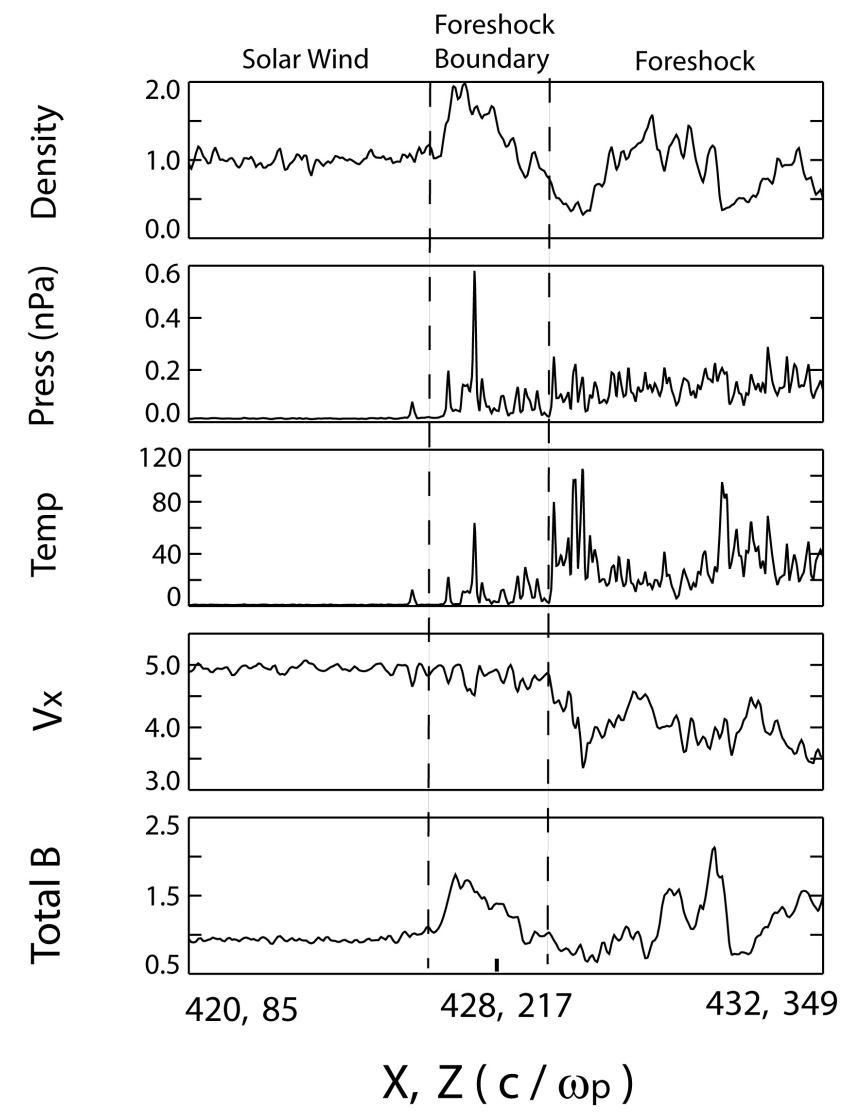

Fig. 2. Densities, velocities, temperatures, and total magnetic field strengths along the vertical cut shown in Fig. 1, from the pristine solar wind (on the left) to the foreshock on the right. Compared to the solar wind, the foreshock is a region of depressed, variable, densities, velocities, and magnetic field strengths, but enhanced temperatures. The boundary of the foreshock is a region of enhanced densities and magnetic field strengths.

As illustrated in Fig. 1, the magnetic field strength and density enhancements associated with this ion foreshock boundary extend far upstream from $X=0$ to $600 c / \omega_{p}$ near $Z=200 \mathrm{c} / \omega_{p}$. Both the width of the boundary and its strength diminish with distance upstream. Figure 3 shows the variation of the density, north-south component of the flow velocity, and magnetic field strength within the boundary as a function of distance upstream from the bow shock along the cut marked by a horizontal dashed yellow line in Fig. 1. By a distance of $550 \mathrm{c} / \omega_{p}$ (corresponding to $\sim 8.6 R_{E}$ ) upstream from the bow shock, the magnetic field strengths and densities have almost fallen to background solar wind values. The persistent southward flow deflection within the boundary is consistent with the predicted southward expansion of this boundary in response to the imbalance between the enhanced pressures of the foreshock to the north and the lower pressures in the pristine solar wind to the south. Just outside the bow shock, $V z / V x$ is $1.4 / 5$, indicating a peak deflection of $\sim 15^{\circ}$.
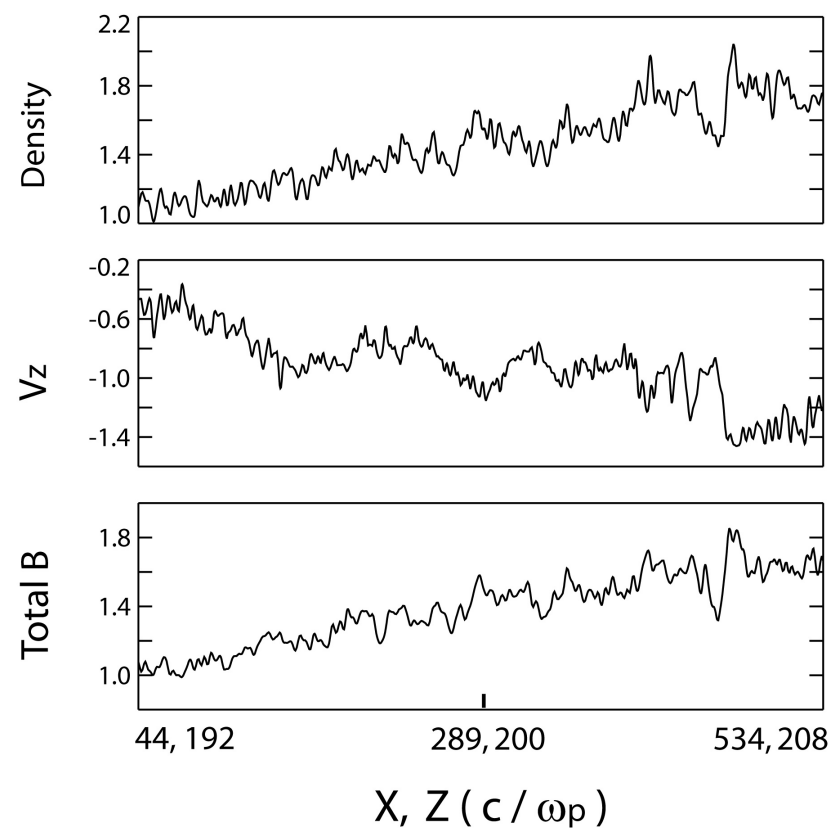

Fig. 3. Densities, magnetic field strengths, and the north/south component of the velocity along the radial dashed line shown in Fig. 1. Densities and magnetic field strengths decrease with distance upstream from the bow shock, while flows are deflected southward.

\section{Comparison with observations}

Sibeck et al. (2002) presented a case study of Wind plasma and magnetic field observations of an isolated 'cavity' that exhibited a hot tenuous core region with weak magnetic fields bounded by density, magnetic field strength, and slight ion temperature enhancements. Events like this are common in the IMP-8, Geotail, Interball-1, and Wind databases (e.g. Sibeck et al., 2004). As heated plasma and energetic ions are invariably observed within the core region of such events, but not outside, the events cannot be density depletions embedded within the foreshock. Nor can they be hot flow anomalies or density holes, for they are not centered on IMF discontinuities and do not exhibit greatly deflected flows. Instead, they are much more easily explained in terms of brief crossings from the pristine solar wind into the foreshock and then back into the solar wind.

Here we compare Cluster plasma and magnetic field observations of the boundary of the foreshock with the predictions of the hybrid code model. Figure 4 presents Cluster 1 and 4 ion (Reme et al., 2001) and electron (Johnstone et al., 1997) plasma and magnetic field (Balogh et al., 2001) observations of the pristine solar wind, foreshock boundary, and foreshock at 8,4 , and $4 \mathrm{~s}$ time resolution respectively during a $100 \mathrm{~min}$ interval on 2 April 2004. During this interval, Cluster 1 and 4 moved from GSE $(\mathrm{x}, \mathrm{y}, \mathrm{z})=(11.2,-10.5,-9.4)$ to $(10.1$, $-10.1,-9.9) R_{E}$ upstream from the southern pre-noon bow shock. At no time were the spacecraft separated by more 


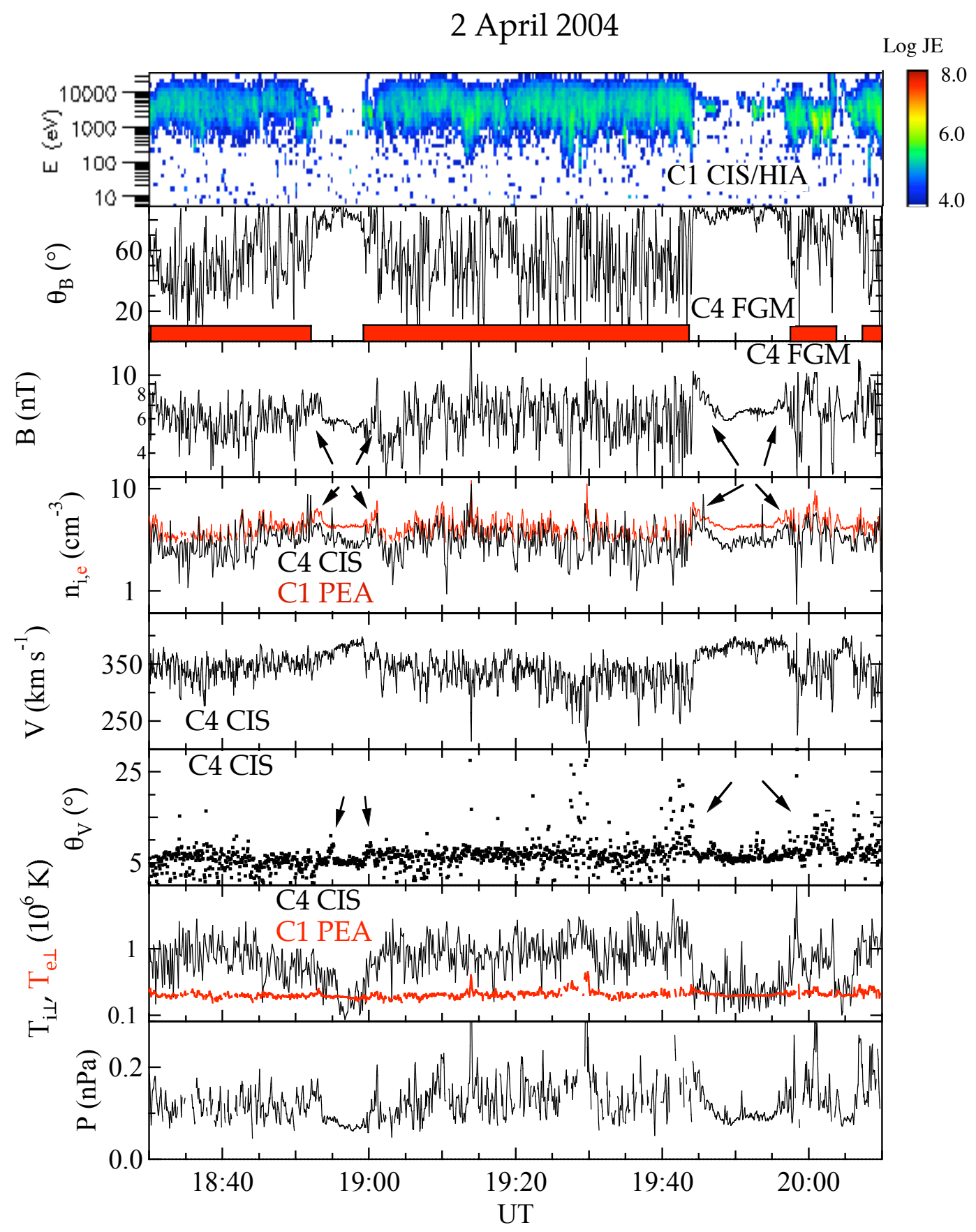

Fig. 4. Cluster-1 and -4 plasma and magnetic field observations from 18:30 to 20:10 UT on 2 April 2004. From top to bottom, the figure displays the CIS/HIA ion energy flux $\left(\mathrm{keV} \mathrm{cm}^{2} \mathrm{~s}^{-1} \mathrm{kev}^{-1}\right)$ arriving at the spacecraft from the full range of meridional look directions and the azimuthal quadrant centered on the antisunward look direction, the cone angle $\left(\theta_{B}\right)$ between the IMF and the Sun-Earth line, the total magnetic field strength, the PEACE electron (red) and CIS/CODIF ion (black) densities, the CIS/CODIF ion flow velocity, the cone angle $\theta_{V}$ between the CIS/CODIF ion velocity and the Sun-Earth line, the PEACE electron (red) and CIS/CODIF ion (black) temperatures, and the total pressure (sum of the magnetic, CIS/CODIF ion thermal, and PEACE electron thermal temperatures). Horizontal red bars in the cone angle panel indicate foreshock intervals marked by low cone angles, enhanced suprathermal ion energy fluxes, depressed plasma flow velocities, and enhanced wave activity. Arrows indicate enhanced magnetic field strengths and densities, and flow deflections, on the edges of the foreshock. Here CIS: Cluster Ion Spectrometry, HIA: Hot Ion Analyzer, CODIF: Composition Distribution Function, PEACE: Plasma Electron and Current Experiment, and FGM: fluxgate magnetometer. 
than $0.05 R_{E}$, i.e. they were essentially collocated for our purposes.

Located very close to the bow shock, the Cluster spacecraft should lie within the foreshock for all but the largest IMF cone angles. Ion foreshock intervals can be identified on the basis of correlated $30 \mathrm{~s}$ period density and magnetic field strength fluctuations and suprathermal $(>1 \mathrm{keV})$ ion fluxes (Fairfield et al., 1990; Eastwood et al., 2006). Based on these considerations, the observations shown in Fig. 4 indicate that Cluster 1 and 4 were within the ion foreshock prior to 18:55, from 19:00 to 19:44, 19:57 to 20:04 and after 20:06 UT. The two spacecraft were within the pristine solar wind from 18:55 to $19: 00,19: 44$ to $19: 57$, and 20:04 to 20:06 UT. As expected, IMF cone angles were less than $80^{\circ}$ during the foreshock intervals, and nearly $90^{\circ}$ during the pristine solar wind intervals.

As predicted by the simulation, Cluster observed enhanced densities and magnetic field strengths on the edges of the foreshock, where the cone angle lies between 80 and $90^{\circ}$ (see arrows in these two panels). Note that partial saturation effects in the time-of-flight ion mass spectrometer depress ion densities in the pristine solar wind, but not the foreshock. The component of the solar wind velocity along the SunEarth line was depressed $\sim 10 \%$ within the foreshock, while ion (but not electron) temperatures were greatly enhanced. Arrows in the flow deflection panel indicate that flows on the edges of the foreshock were deflected away from the antisunward direction. Flows were also deflected from this direction deep within the foreshock when and where the solar wind was greatly decelerated (e.g. 19:30 UT). Finally, as predicted, the total pressure was far greater within the foreshock than in the pristine solar wind.

Figure 5 compares scatter plots for the magnetic field strength, ion velocity, ion and electron temperatures versus density in the simulation (left column) and the Cluster observations (right column). The data points are taken from within the box shown in Fig. 1 and the interval of Cluster observations shown in Fig. 4. The simulation predicts the linear relationship between the magnetic field strength and density seen during foreshock ULF wave intervals and crossings through the edge of the foreshock. It predicts the tendency for high densities to be associated with high velocities, but low densities to be associated with a broad range of depressed velocities. Finally, it also predicts the inverse relationship between the ion temperature and the density. Because the hybrid model treats the electrons as a fluid, their temperature can be determined from the density and the polytropic gas law. An assumption concerning the polytropic index is needed. A comparison of the bottom panels in Fig. 5 reveals that the observed electron temperature variations (right column) are more nearly isothermal than adiabatic, i.e. better explained by a polytropic index $\gamma$ of 1.1 than one of 1.666 , consistent with values for the solar wind as a whole (Sittler and Scudder, 1980).
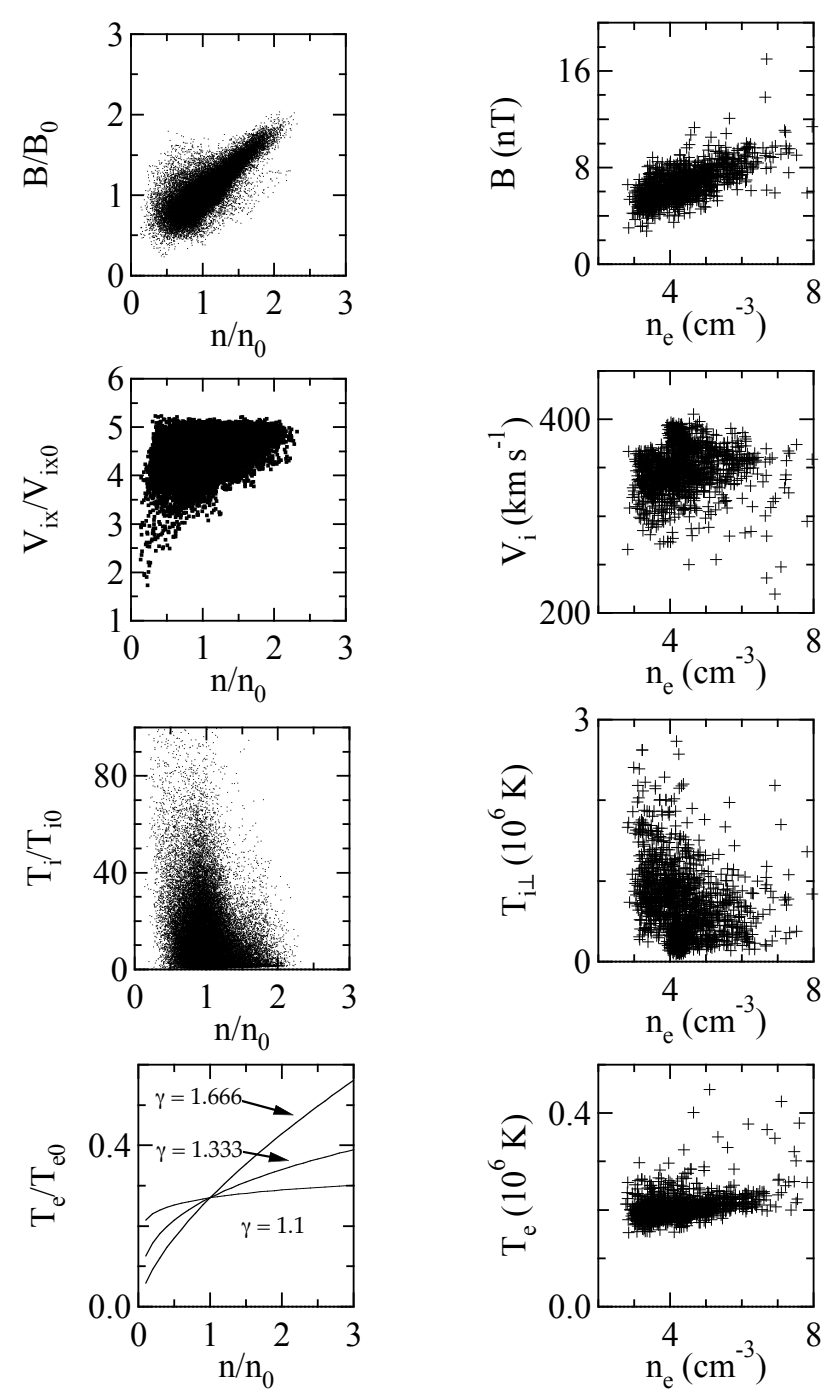

Fig. 5. Scatter plots of velocity, temperature, and magnetic field strength versus density for observations within the box shown in Fig. 1 and the Cluster observations shown in Fig. 4.

\section{Summary and conclusion}

The edge of the foreshock is the boundary between IMF lines with and without backstreaming ions. Suprathermal ions contribute significantly to the sum of the thermal and magnetic pressures within the foreshock. The imbalance between the enhanced pressures within the foreshock and the pristine solar wind causes the former region to expand at the expense of the latter. As the foreshock expands, it displaces and compresses the neighboring solar wind plasma and magnetic field, creating an edge region of enhanced plasma densities and magnetic field strengths.

Both the strength of the perturbations within the edge and its width diminish with distance upstream, just as in actual observations (e.g. Sibeck et al., 2004). According to the simulation results presented in this paper, the densities within the 
edge and just upstream from the bow shock can be enhanced by as much as a factor of 2 and magnetic field strengths by as much as a factor of 1.7. Within the edge, flow velocities are slightly deflected away from the foreshock towards the pristine solar wind, consistent with the expected expansion of the foreshock.

Solar wind parameters constantly vary. Transient rotations in the IMF orientation can cause the edge of the foreshock to pass over a spacecraft and then return to its original position. As a result, spacecraft will make transient passages from the pristine solar wind through the ion foreshock boundary and into the foreshock, followed by returns into the pristine solar wind. During these encounters, the spacecraft will observe hot tenuous plasmas with weak magnetic field strengths bounded by enhanced densities and magnetic field strengths. Such features have been reported on numerous occasions. The results presented here provide a satisfactory explanation for the foreshock cavities in terms of spacecraft encounters with the ion foreshock boundary.

We compared the predictions of the hybrid simulation with observations. The code accurately predicts the inverse relationship between densities and ion temperatures during encounters with the foreshock and its boundary. It correctly predicts the depressed densities and broad range of depressed velocities within the foreshock. The enhanced temperatures and corresponding pressures within the foreshock reduce densities and magnetic field strengths, resulting in a simple linear correlation between the latter two parameters during ULF wave intervals and crossings of the foreshock boundaries. Electrons remain nearly isothermal, with a polytropic index similar to that observed in the pristine solar wind.

Acknowledgements. Work at Solana Scientific was supported by grants from NSF \# ATM-0502992 and NASA \# NNX06AD78G. Work at GSFC was supported by NASA's Guest Investigator program.

Topical Editor I. A. Daglis thanks G. Zastenker and C. Mazelle for their help in evaluating this paper.

\section{References}

Balogh, A., Carr, C. M., Acuña, M. H., et al.: The Cluster Magnetic Field Investigation: overview of in-flight performance and initial results, Ann. Geophys., 19, 1207-1217, 2001,

http://www.ann-geophys.net/19/1207/2001/.

Eastwood, J. P., Lucek, E. A., Mazelle, C., Meziane, K., Narita, Y., Pickett, J., and Treumann, R. A.: The foreshock, Space Sci. Rev., 11, 41-94, 2006.
Fairfield, D. H., Baumjohann, W., Paschmann, G., Lühr, H., and Sibeck, D. G.: Upstream pressure variations associated with the bow shock and their effects on the magnetosphere, J. Geophys. Res., 95, 3773-3786, 1990.

Johnstone, A. D., Alsop, C., Burge, S., et al.: Peace: a plasma electron and current experiment, Space Sci. Rev., 79, 351-398, 1997.

Lin, Y.: Global hybrid simulation of hot flow anomalies near the bow shock and in the magnetosheath, Planet. Space Sci., 50, 577-591, 2002.

Lin, Y.: Global-scale simulations of foreshock structures: at the quasi- parallel bow shock, J. Geophys. Res., 108, 1390, doi:10.1029/2003JA009991, 2003.

Omidi, N., Blanco-Cano, X., Russell, C. T., and Karimabadi, H.: Dipolar magnetospheres and their characterization as a function of magnetic moment, Adv. Space Res., 33, 1996-2003, 2004.

Omidi, N., Blanco-Cano, X., and Russell, C. T.: Macrostructure of collisionless bow shock: 1. Scale lengths, J. Geophys. Res., 110, A12212, doi:10.1029/2005JA011169, 2005.

Omidi, N.: Formation of cavities in the foreshock, in: Turbulence and Nonlinear Processes in Astrophysical Plasmas, edited by: Dastgeer, D. and Zank, G. P., AIP conference proceedings, vol. 932, 181, 2007.

Parks, G. K., Lee, E., Mozer, F., et al.: Larmor radius size density holes discovered in the solar wind upstream of Earth's bow shock, Phys. Plasmas, 13, 050701, 2006.

Rème, H., Aoustin, C., Bosqued, J. M., et al.: First multispacecraft ion measurements in and near the Earth's magnetosphere with the identical Cluster ion spectrometry (CIS) experiment, Ann. Geophys., 19, 1303-1354, 2001, http://www.ann-geophys.net/19/1303/2001/.

Schwartz, S. J., Paschmann, G., Sckopke, N., et al.: Conditions for the formation of hot flow anomalies at the Earth's bow shock, J. Geophys. Res., 105, 12 639-12 650, 2000.

Sibeck, D. G., Baumjohann, W., Elphic, R. C., et al.: The magnetospheric response to 8-min-period strong amplitude upstream pressure variations, J. Geophys. Res., 94, 2505-2519, 1989.

Sibeck, D. G., Phan, T.-D., Lin, R., Lepping, R. P., and Szabo, A.; Wind observations of foreshock cavities: A case study, J. Geophys. Res., 10, 1271, doi:10.1029/2001JA007539, 2002.

Sibeck, D. G., Kudela, K., Mukai, T., Nemecek, Z., and Safrankova, J.: Radial dependence of foreshock cavities: A case study, Ann. Geophys., 22, 4143-4151, 2004, http://www.ann-geophys.net/22/4143/2004/.

Sittler Jr., E. C. and Scudder J. D.: An empirical polytrope law for solar wind thermal electrons between 0.45 and $4.76 \mathrm{AU}$ : Voyager 2 and Mariner 10, J. Geophys. Res., 85, 5131-5137, 1980.

Thomas, V. A. and Brecht, S. H.: Evolution of diamagnetic cavities in the solar wind, J. Geophys. Res., 93, 11 341-11 353, 1988.

Wibberenz, G., Zöllich, F., Fischer, H. M., and Keppler, E.: Dynamics of intense upstream ion events, J. Geophys. Res. 90, 283-301, 1985. 